\title{
Les psychotropes haute couture
}

\author{
Designer drugs
}

Marc DEVEAUX, Didier GOSSET

Institut de Médecine Légale, Place Théo Varlet - 59000 LILLE - Tél : 0320621223 - Fax : 0220621229 e-mail : mdeveaux@easynet.fr

(Reçu le 17 décembre 1999 ; accepté le 19 janvier 2000)

\section{$R \hat{E} S U M E ́$}

Un designer est celui qui conçoit des objets usuels, industriels ou urbains, tendant à harmoniser l'environnement humain. Ce terme américain appliqué à des substances psychoactives définit la synthèse de toute une série de psychotropes fabriqués clandestinement, les designer drugs. La définition du dictionnaire est donc redoutable quand elle s'applique à des substances chimiques illégales destinées à améliorer les performances : ainsi l'environnement humain serait harmonisé. C'est pourquoi nous lui préférons les termes de "psychotropes haute couture" ou "cousus mains", puisque ces produits sont fabriqués quasiment pour créer la mode ou la suivre de très près et s'adapter ainsi aux marchés clandestins.

"Adam", "Eve", "ecstasy", "china white", "ice" sont les produits les plus connus dont on peut user et abuser pour tenter d'accrô̂tre ses performances en utilisant leurs propriétés psychotropes, notamment entactogènes. Nous décrivons les différentes familles chimiques et leurs principaux effets pharmacologiques et toxiques, les voies de synthèse susceptibles d'être utilisées par les laboratoires clandestins, les dangers de ces synthèses.

Les méthodes d'analyse et d'identification des psychotropes haute couture doivent être très performantes: chromatographie en phase gazeuse couplée à la spectrométrie de masse, spectroscopie $R M N d u^{13} \mathrm{C}$ et du ${ }^{1} \mathrm{H}$, électrophorèse capillaire avec des séparateurs chiraux, chromatographie liquide haute pression couplée au dichroisme circulaire et la plus spécifique, la spectroscopie infrarouge par transformée de Fourier couplée ou non à la chromatographie en phase gazeuse. Les impuretés constituent souvent la signature du fabriquant et il est indispensable de les identifier. Malheureusement ces drogues ne sont parfois identifiées que tardivement et avec difficultés par les toxicologues analystes. MOTS-CLÉS

Designer drug, amphétamines, synthèse, analyse.

\section{SUMMARY}

The term "designer drug" may refer to illegaly synthetised psychoactive drugs or to drugs dedicated to physical performances' increasing. However, those substances can be called "haute couture drugs" since they are clandestinaly synthetised and marketed in order to be fully fashioned or to create the fashion.

Adam, Eve, Ecstasy, ChinaWhite, Ice are the best known, can be used on a continuous basis and are drugs of abuse. We describe the different groups of drug and there main pharmacological properties and toxicity. Miscellanous laboratory and clandestine syntheses are given, as well as the potential dangers of illicit manufacturing.

Analysis of seizure designer drugs must be done with high technology apparatus : GC-MS, ${ }^{13} \mathrm{C}$ - and ${ }^{1} \mathrm{H}-\mathrm{NMR}$, capillary electrophoresis with chiral separation, HPLC-circular dichroism, Fourier transform infrared spectrometry alone or coupled with gas chromatography. Identification of the major impurities may constitute a fingerprint of the batch of the illicit drugs.

\section{KEY-WORDS}

Designer drugs, amphetamines, synthesis, analysis. 


\section{Introduction}

Le terme designer [di'zajnoer] est un américanisme définissant le spécialiste de la conception d'objets industriels, urbains ou usuels tendant par leur esthétisme et leur nouveauté à harmoniser l'environnement humain, en les adaptant particulièrement à leur fonction. Appliqué à des substances psychoactives depuis les années 1960, ce mot définit toute une série de psychotropes fabriqués clandestinement, les designer drugs (1). La définition du dictionnaire est donc redoutable quand elle s'applique à des substances chimiques illégales destinées à améliorer les performances : l'environnement humain serait ainsi harmonisé. C'est pourquoi nous lui préférons le terme de psychotropes "haute couture" ou psychotropes "cousus main", puisque ces produits sont fabriqués artisanalement, quasiment sur mesure pour créer ou suivre la mode et s'adapter ainsi aux marchés clandestins (2). Cependant tous ces vocables seront utilisés ici indifféremment. Ils ne s'appliquent qu'à des substances : (a) synthétisées clandestinement à partir de produits chimiques faciles à se procurer, (b) n'entrant dans le cadre d'aucune réglementation, ou (c) qui sont des copies clandestines de psychotropes commercialisés, (d) vendues clandestinement sous des noms le plus soyvent exotiques et attirants et (e) utilisées dans un but récréatif (1). Ces substances peuvent induire une toxicomanie.

Il ne faut donc pas les confondre avec les substances issues de la recherche pharmaceutique, où la coopération des chimistes et des pharmacologues aboutit, après des recherches exhaustives et systématiques sur des centaines de produits, à la commercialisation de quelques médicaments actifs. La série des ß-bloquants est un des plus beaux exemples (3). Le sildénafil en est un autre (4). Par extension, on qualifie aussi de designer drug des produits anciens auxquels on découvre de nouvelles propriétés (5) ou des produits nouveaux à la recherche de leur cible cellulaire ou biochimique exacte (6).

\section{Les principaux produits}

On distingue deux grands groupes, les opioïdes et les phényléthylamines $(3,7)$. Nous nous intéresserons essentiellement à ces dernières. Les plus connues sont regroupées dans le tableau $\mathrm{I}$.

\section{Les opioïdes}

Le fentanyl est le chef de file d'un groupe d'opioïdes synthétiques issus de la recherche des laboratoires Janssen. Sa durée d'action est très courte et il est environ 80 fois plus puissant que la morphine. Aux ÉtatsUnis, le fentanyl était utilisé comme analgésique depuis 1968, quand en 1979 est apparu sur le marché clandestin l' $\alpha$-méthyl-fentanyl, appelé "China white" ou "héroïne synthétique". Dix ans après, il avait causé la mort d'une centaine de personnes $(1,8,9)$. Mille fois plus actif que la morphine, il est consommé par voie intraveineuse ou transdermique, plus rarement fumé,

Tableau I : Différents psychotropes "haute couture". Deux molécules différentes peuvent porter le même nom vernaculaire. Les doses efficaces sont données à titre indicatif (16).

\begin{tabular}{|l|c|l|l|}
\hline Abbréviation & Dose & Dénomination chimique internationale & Noms courants \\
\hline AMF & $1 \mu \mathrm{g}$ & alpha-méthylfentanyl & China white, héroïne synthétique \\
\hline 3MF & $1 \mu \mathrm{g}$ & 3-méthylfentanyl & \\
\hline MA & $50 \mathrm{mg}$ & méthamphétamine & Speed, Ice \\
\hline MDMA & $\begin{array}{l}150 \mathrm{à} \\
200 \mathrm{mg}\end{array}$ & 3,4 -méthylènedioxyméthamphétamine & $\begin{array}{l}\text { Ecstasy, XTC, Xtasy, Adam, E, ecsta, M\&M, } \\
\text { Eckie, MDM }\end{array}$ \\
\hline MDA & $150 \mathrm{mg}$ & 3,4 -méthylènedioxyamphétamine & $\begin{array}{l}\text { Love drug, Love bug, Love, Hug drug, Meth, Ice, } \\
\text { Amp, Crystal, Crank, Speed, Whizz, Snowball }\end{array}$ \\
\hline MDEA & $100 \mathrm{mg}$ & 3,4-méthylènedioxythamphétamine & MDE, Eve \\
\hline MBDB & $200 \mathrm{mg}$ & n-méthyl-1-(1,3-benzodioxol-5yl))-2-butanamine & Eden, Méthyl J \\
\hline BDB & $200 \mathrm{mg}$ & 1-(1,3-benzodioxol-5-yl)-2-butanamine & J \\
\hline DOB & $1 \mathrm{mg}$ & 4-bromo-2,5-diméthoxyamphétamine & Acid, LSD, Nexus \\
\hline DOM & $3-10 \mathrm{mg}$ & 4-méthyl-2,5-diméthoxyamphétamine & STP (serenity, tranquility, peace) \\
\hline PMA & $50 \mathrm{mg}$ & para-méthoxyamphétamine & 4-MA \\
\hline MTA & & para-méthylthioamphétamine & S-5 \\
\hline méthcathinone & & méthylamino-1-phénylpropan-1-one & Cat, Jeff, Ephédrone, Goop, Mulka \\
\hline 2C-B & $10-20 \mathrm{mg}$ & 4-bromo-2,5-diméthoxyphénéthylamine & Bromo, Nexus \\
\hline aminorex & & 2-amino-5-phényl-delta -2oxazoline & \\
\hline 4-MAX & dl-cis-4-méthylaminorex & U4Euh, Euphoria, McN-822, Ice \\
\hline
\end{tabular}


sniffé ou par voie orale. Un autre dérivé, le cis-3méthyl-fentanyl (6000 fois plus actif que la morphine) a été vendu aux Etats-Unis sous les noms de "Mexican brown" ou de "Persian white". Une dizaine d'autres dérivés dont le parafluorofentanyl et le thienylfentanyl ont été décrits, avec une fréquence d'utilisation variable selon les années $(1,10)$. L'usage des analogues du fentanyl en Europe semble encore rare, mais peut-être estce du à la difficulté de leur mise en évidence (11) ?

En 1981, des chimistes clandestins à la recherche d'analogues de la mépéridine (péthidine) synthétisèrent de la MPPP (1-méthyl-4-phényl-propionoxypipéridine) qui contenait une impureté, la MPTP (1-méthyl-4-phényl-1,2,3,6-tétrahydropyridine) (12). On s'est aperçu rapidement que la MPTP pouvait être à l'origine d'une véritable maladie de Parkinson expérimentale (13).

Ces opioïdes sont des analgésiques puissants, avec une composante tranquillisante et qui, administrés par voie intraveineuse, procurent une euphorie semblable à celle de l'héroïne. Les symptômes de l'intoxication aiguë sont identiques à ceux causés par les opiacés.

\section{Les phényléthylamines}

Dès 1947, Simonin (14) introduisait le vocable de "vigilamine" dans son cours de médecine légale toxicologique. C'était la traduction du mot allemand Weckamine ou amine du réveil, de l'éveil. On parle aujourd'hui, comme Laure (15), de gélules de la performance. Ces substances sont des dérivés de la phénéthylamine et Alexander Shulgin en a synthétisé et testé sur lui même ou fait testé presque 200 dérivés (16) : l'acronyme qui donne le titre à son livre longtemps introuvable se lit "Phenethylamines I Have Known And Loved". Ce sont ces phénéthylamines et leurs dérivés qui sont les véritables designer drugs. Parallèlement, il a aussi développé une série de 55 tryptamines aux propriétés hallucinogènes. Mais c'est une autre histoire (17).

Ces produits sont connus depuis le début du siècle et furent utilisés pour leurs propriétés stimulantes pendant les deux guerres mondiales, puis comme anorexigènes $(3,14,18)$. A partir des années 60 , leur vente et leur utilisation furent sévèrement réglementées dans les pays occidentaux. Dans les années 70-80, les propriétés entactogènes et hallucinogènes de certains dérivés ont été mises en exergue et ces designer drugs ont alors envahi les marchés clandestins : c'est l'avènement de la MDA, de la MDEA, de la MDMA. On peut classer les principales molécules en trois grandes catégories, selon leur action :

\section{Phényléthylamines stimulantes}

Ce sont l'amphétamine et la méthamphétamine, essentiellement utilisées comme dopants par les sportifs, les étudiants, les cadres pour "donner du nerf aux gens fatigués", car "deux pilules valent mieux qu'un mois de vacances" (19). Peu toxiques par voie orale aux doses habituelles, elles le sont éminemment plus si les doses sont très augmentées ou répétées, ou encore si l'administration se fait par voie intraveineuse.

On peut ajouter ici des produits ayant des effets stimulants, anorexigènes et hypertenseurs, dérivés de la phénylisopropylamine. Il s'agit de l'aminorex, du 4-méthylaminorex et de la méthcathinone $(20,21)$. Des intoxications mortelles avec une très forte hypertension pulmonaire ont été décrites $(22,23)$.

\section{Phényléthylamines entactogènes}

Ce sont des dérivés 3,4 méthylènedioxy-. On retrouve la MDA (méthylènedioxyamphétamine), la MDMA (méthylènedioxyméthamphétamine), la MDEA (méthylènedioxyéthamphétamine), la BDB (1-(1,3benzodioxol-5-yl)-2-butanamine) et la $\mathrm{MBDB}$ (son dérivé $\mathrm{N}$-méthylé). Actives per os, ces molécules facilitent les contacts et le rapprochement des individus par une exacerbation des sensations psychiques et tactiles (définition de l'effet entactogène proposée par Nichols en 1986 (24)). Les effets sont agréables ou désagréables selon les individus. Le risque d'accoutumance physique est presque nul, mais la dépendance psychique est très sévère. La toxicité somatique directe à court terme est importante : tachycardie, hypertension, hyperthermie, collapsus par déshydratation. Il faut aussi compter sur les effets toxiques indirects induits par les comportements à risques qu'elles induisent. Longtemps méconnus, les troubles neurobiologiques dus à la consommation de la MDMA peuvent être graves : troubles anxio-dépressifs, insomnie, problèmes cognitifs, épisodes de confusion mentale voire de décompensation d'allure paranoïde (25-30). Les décès par surdosage ne sont pas rares (31-33). Le rapport d'un récent voyage ethnographique montre qu'en France l'ecstasy "s'est maintenant infiltrée chez les 15-25 ans au même titre que le tabac, l'alcool et le cannabis, et s'inscrit dans le mouvement culturel des nouvelles musicales (house, techno, rave-parties, Tecknival)" (34). On retrouve les phénétylamines entactogènes dans toute l'Europe (25, 35-42), l'Asie (43), l'Australie (3).

\section{Phényléthylamines hallucinogènes}

Les plus connues sont la DOB (4-bromo-2,5-diméthoxyamphétamine), la DOM ou STP (4-méthyl-2,5diméthoxyamphétamine), la TMA (2,4,5-triméthoxyamphétamine), la PMA (paraméthoxyamphétamine), le 2C-B (4-bromo-2,5-diméthoxyphénéthylamine). Ces dérivés di- ou triméthoxylés, analogues de la mescaline produisent à des degrés divers les mêmes effets hallucinogènes, auxquels peuvent s'ajouter des effets sympathomimétiques parfois graves (44). La DOB est la plus active et peut se trouver sur le marché clandestin sous la même forme de buvard que le LSD (13). Sont apparues très récemment des thioamphétamines (déjà synthétisées par Shulgin il y a plusieurs années avec 75 autres dérivés diméthoxylés (16)) : la 2C-T-2 (4-éthyl- 
thio-2,5-diméthoxyphénéthylamine) et la MTA (pméthylthioamphétamine), puissant sérotoninergique (45, Ghysel M.H.(communication personnelle)).

\section{Phényléthylamines inclassables}

Plusieurs dérivés de la 1-phényléthylamine ont été découverts sur le marché clandestin en Europe (1amino-1-(4-méthylphényl)éthane, 1-méthylamino-1phényléthane, 1-phényléthylamine). Leurs effets sont encore inconnus (46-48).

\section{Les principales voies de synthèse et les impuretés de fabrication}

Le but du "grand couturier" (designer) est de fabriquer des analogues de produits soumis à des réglementations strictes, qui puissent être vendus sans enfreindre la loi. Il s'agit donc de pouvoir acheter les ingrédients chimiques de base assez anodins de façon légale pour aboutir à un đérivé "non illégal" au moins aussi actif que le modèle copié. Les synthèses chimiques se font généralement dans des lieux domestiques (cuisine, garage, caravane, conteneur). Les contrôles de pureté et les analyses permettant de libérer les lots sont bien sur inexistants. Au début des années 80, les chimistes clandestins étaient considérés comme très inexpérimentés et utilisaient des réactifs de médiocre qualité. De nombreuses bibliothèques universitaires américaines se plaignent alors de voir leur documentation pillée par les chimistes clandestins : revues et livres de chimie volés, pages arrachées (49).

On ne trouve que peu de détails dans la littérature de toxicologie médicolégale et de criminalistique sur les diverses voies de synthèse des dérivés du fentanyl. Le benzylfentanyl semble être un précurseur souvent utilisé : c'est une impureté retrouvée dans le fentanyl illicite. Les fentanyls dérivés en -4 , utilisés en thérapeutique, sont parfois détournés de leur usage. Ils sont plutôt consommés directement par les toxicomanes que comme précurseurs dans les synthèses (10).

L'histoire la plus édifiante est celle de la MPTP, déjà évoquée plus haut $(3,12)$. Dans le comté de Santa Clara (Californie), un "grand couturier" décida en 1981 de synthétiser un analogue de la péthidine : la MPPP (1méthyl-4-phényl-propionoxypipéridine ou 4-propyloxy4-phényl-N-méthylpipéridine). Afin de gagner du temps et de l'argent, il décida de diminuer les temps de réaction et d'augmenter les températures. Cela permit la synthèse en quantité importante d'une impureté indésirable, la MPTP (1-méthyl-4-phényl-1,2,3,6-tétrahydropyridine), très toxique pour les neurones dopaminergiques.

Les chimistes amateurs et clandestins ont cependant fait des progrès rapides et ont vite appris à synthétiser la plupart des phényléthylamines. C'est donc dans ce domaine que le commerce illicite s'est développé et donc que les saisies sont les plus fréquentes $(40,42)$. II s'est avéré possible, par l'analyse fine des comprimés saisis, d'identifier les voies de synthèse et les produits de départ $(39,49$, Vermeulen T.M. (communication personnelle)). C'est un problème complexe. En effet Shulgin indique par exemple qu'il y a environ vingt façons de synthétiser la MDA (16). On peut partir esentiellement de la pipéronylacétone, mais aussi du pipéronal, du B-nitroisosafrole, du safrole ou de l'iso-safrole. Les racines et l'huile de sassafras ont même été utilisées (49). En France, tous ces produits, utilisés notamment dans l'industrie des parfums, sont soumis à un contrôle en tant que précurseurs chimiques de stupéfiants (décrets 96-1060 et 96-1061 du 5 décembre 1996, arrêté du 10 décembre 1996). Pour faire l'amination réductive d'une cétone (réaction de Leuckart et Wallach), la voie la plus simple et la plus rapide est celle qui utilise la pipéronylacétone $(39,50,51)$. Les principales impuretés peuvent être alors la $N, \alpha, \alpha^{\prime}$-triméthyldiphénétylamine, la phénylméthamphétamine, la méthylamine et des produits de condensation résineux complexes (52). Si l'on veut partir de la phényl-2propanone, il faut la synthétiser. Cette opération, si elle est mal dirigée, conduit également à la synthèse d'alkènes impossibles à séparer du produit fini : c'est la marque de fabrique du laboratoire clandestin (53).

La synthèse d'une amine à partir d'une cétone est tout à fait possible par l'intermédiaire d'une base de Schiff, mais implique des conditions de réduction très strictes (réaction de Birch). On retrouve alors comme impuretés les sels des dérivés aminés de départ et des amines tertiaires, ainsi que l'alcool formé par réduction directe de la cétone de départ en excès. La découverte d'hydrastinine permet de prouver que cette voie de synthèse a été utilisée pour la 3,4-MDMA (54).

La condensation du benzaldéhyde avec un nitroalcane forme un $ß$-nitrostyrène qui sera ensuite réduit par voie catalytique. Les impuretés les plus courantes seront donc les formes réduites de tous les précurseurs (alcools benzyliques pour les aldéhydes, éthylamine pour le nitroéthane). On peut aussi retrouver des nitrostyrènes en excès.

A titre d'exemple la synthèse de la d-méthamphétamine se fait par l'action de l'acide iodhydrique et du phosphore rouge sur la l-éphédrine ou la d-pseudoéphédrine, l'intermédiaire formé étant l'iodométhamphétamine. Les impuretés retrouvées sont : phényl-2-propanone, 1-benzyl-3-méthylnaphtalène, 1,3-diméthyl-2-phénylnaphtalène $(16,55)$. Au cours de la préparation de l'acide iodhydrique par l'action du phosphore rouge sur l'iode, il peut se dégager de l'acide phosphorique qui se décompose au dessus de $180^{\circ} \mathrm{C}$ en donnant de l'hydrogène phosphoré : dans les laboratoires clandestins où la température de réaction n'est pas bien régulée, ce phé- 
nomène peut se produire facilement et les intoxications mortelles des designers ne sont pas rares (56). Les autres réactifs tels que l'ammoniac anhydre, le chlorure d'ammonium et le sodium métal sont dangereux et les enquêteurs doivent être prudents lors de l'exploration des laboratoires clandestins (57).

Les phénéthylamines bromées sont fabriquées plutôt par bromation que par réduction à partir de leurs homologues.

\section{Méthodes physico-chimiques d'identification}

Toutes les méthodes physico-chimiques peuvent être utilisées pour séparer et identifier les différents composant de comprimés de designer drugs. Bien entendu les méthodes les plus modernes doivent être privilégiées. Nous en donnerons quelques exemples.

\section{Méthodes physiques}

Julian a étudié et répertorié les cristaux que les amphétamines hallucinogènes forment avec l'acide 5-nitrobarbiturique (58), mais il concède que cette technique ne peut intervenir qu'en complément des méthodes spectroscopiques. Elle reste néanmoins simple et bon marché. On peut utiliser les réactions avec le chlorure de platine et le chlorure d'or pour les dérivés de l'aminorex (20).

Si un produit saisi semble pur, la mesure du point de fusion et celle du pouvoir rotatoire sont également de bons éléments d'orientation.

\section{Chromatographie en phase gazeuse- spectrométrie de masse (CG/SM)}

La présence de carbones asymétriques implique l'existence de stéréoisomères, dont certains sont psychoactifs et d'autres non. Les produits vendus sur le marché clandestin ne sont jamais purs : la différenciation des isomères est donc importante dans la démarche d'identification des comprimés. Il faut aussi se souvenir que le métabolisme est stéréospécifique et que les isomères devront également être recherchés dans les liquides biologiques. La séparation de la MDA, MDMA et MDEA se fait sur une colonne capillaire DB-1 ou DB$5(2,53)$. Tous les intermédiaires de la synthèse de la méthamphétamine à partir de la phényl-2-propanone ont déjà été séparés et identifiés par CG/SM sur une colonne à $5 \%$ de phénylméthylsilicone (59). On peut très facilement utiliser une colonne chirale $(60)$ ou de faire un dérivé chiral avant l'injection (61). Cependant, les diastéréoisomères des intermédiaires de synthèse du 4-méthylaminorex doivent être suffisamment séparés pour être identifiés sur des colonnes classiques (20).

\section{Chromatographie liquide haute pression (CLHP)}

Il est tout à fait possible de séparer les différentes amphétamines par CLHP avec une détection par spectrométrie ultraviolet, mais l'essentiel tient dans l'identification des stéréoisomères. Différentes techniques sont utilisables : dérivation chirale puis analyse, utilisation d'une phase stationnaire chirale, ou encore addition d'un agent chiral à la phase mobile. Une revue complète du problème a été proposée récemment par Jirovsky (62). Le mode de détection n'est pas toujours classique : SM ou SM/SM, dichroïsme circulaire (63). La simplicité de ce dernier type de détecteur le rend très attrayant, mais il ne semble pas assez sensible.

\section{Résonance magnétique nucléaire (RMN)}

L'identification des methoxyamphétamines par RMN du ${ }^{13} \mathrm{C}$ date des années 80 (64), mais tous les isomères ne pouvaient pas être distingués. Il est possible maintenant en une opération de prendre une empreinte des composants organosolubles. L'interprétation des spectres peut être automatisée et d'énormes progrès ont été réalisés dans les logiciels d'interprétation $(65,66)$. La spectrométrie RMN du proton a aussi été appliquée avec succès à l'identification du 4-méthylaminorex (23).

\section{Electrophorèse capillaire $(\mathbf{E C})$}

Grâce à sa rapidité et sa grande résolution, l'EC est de plus en plus employée pour l'analyse de drogues saisies (67). Les détecteurs vont du simple spectromètre ultraviolet au spectromètre de masse (SM et SM/SM), de façon à améliorer la sensibilité. Des revues entières sont consacrées à l'analyse des amphétamines par CE et une revue générale vient d'être publiée (68).

\section{Spectrométrie infrarouge}

La spectrométrie infrarouge présente l'avantage sur la spectrométrie de masse de permettre l'identification des différents isomères des amphétamines. Toutes les ressources de la spectrométrie infrarouge ont déjà été utilisées : la confection d'un disque avec du $\mathrm{KBr}$ et la prise $\mathrm{du}$ spectre infrarouge par transformée de Fourier (IRTF) ne permet pas l'identification de l'amphétamine suspecte, puisqu'elle n'est pas pure, mais permet la comparaison entre différents lots saisis. On réalise ainsi des profils de comprimés. Le couplage CG/IRTF autorise la prise de spectres très rapidement (moins d'une seconde). La comparaison des spectres obtenus avec ceux d'une bibliothèque informatisée est devenu un outil extrêmement puissant pour l'identification de tous les composés organiques d'un comprimé $(57,69)$.

Enfin, des appareils portables travaillant dans le proche infrarouge permettent de distinguer très rapidement la MDE de la MDMA et de l'amphétamine, en pratiquant des analyses non destructives $(70,71)$. La spectrométrie IRTF est actuellement la méthode d'identification la plus puissante. 


\section{Conclusion}

Les psychotropes haute couture (designer drugs) sont présentés maintenant au public comme des substances de la performance : danser plus longtemps, attirer plus facilement, conduire sans fatigue apparente (72). Mais de l'usage récréatif, on passe vite à l'usage intensif et à la recherche d'autres substances plus efficaces et surtout restant dans le champ de la légalité. Il s'est donc développé un marché parallèle allant de la fabrication à la vente, où des designers suivent ou devancent la mode. Les toxicologues ont maintenant à leur disposition de puissants outils d'analyse leur permettant de cerner les flux de marchandises saisies, essentiellement en établissant des comparaisons de compositions centésimales : le profiler poursuit le designer.

\section{Références}

1. Henderson G.L. Designer drugs : past history and future prospects. J. Forensic Sci. 1988 ; 33 (2) : 569-75.

2. Bost R.O. 3,4-Methylenedioxymethamphetamine (MDMA) and other amphetamine derivatives. J. Forensic Sci. $1988 ; 33$ (2) : 576-87.

3. Chesher G. Designer drugs-The "whats" and the "whys". Med. J. Aus. $1990 ; 153: 157-61$.

4. Guirguis W.R. Oral treatment of erectile dysfunction : from herbal remedies to designer drugs. J. Sex. Mar. Ther. $1998 ; 24: 69-73$.

5. Schussheim D.H., Schussheim A.E. Is digoxin a designer oestrogen ? Lancet $1998 ; 351: 1734$.

6. Rifkind B.M., Rossouw J.E. Of designer drugs, magic bullets, and gold standards. JAMA $1998 ; 279$ (18) : 1483-5.

7. Sternbach G.L., Varon J. "Designer drugs". Recognizing and managing their toxic effects. Postgrad. Med. 1992;91 (8) : 169-76.

8. Zyporin T. A growing industry and menace : makeshift laboratory's designer drugs. JAMA $1986 ; 256$ (22) : 3061-3.

9. Poklis A. Fentanyl : a review for clinical and analytical toxicologists. Clin Toxicol.1995; 33: 439-47.

10.Henderson G.L. Fentanyl-related deaths : demographics, circumstances, and toxicology of 112 cases. J. Forensic Sci. $1991 ; 36(2): 422-33$.

11.Berens J.L., Voets A.J., Demedts P. Illicit fentanyl in Europe. Lancet 1996, 347 : 1334-5.

12.Weingarten H.L. 1-methyl-4-phenyl-1,2,3,6-tetrahydropyridine (MPTP) : one designer drug and serendipity. J. Forensic Sci. 1988 ; 33 (2) : 588-95.

13.Langston J, Ballard P., Tetrud J. Chronic parkinsonism in humans due to a product of meperidine-analog synthesis. Science, $1983 ; 219: 979-80$.

14.Simonin C. Médecine légale judiciaire, Maloine, Paris, 1947, pp.568-569.

15.Laure P. Les gélules de la performance, Ellipses, Paris, 1997, pp.11-30.

16.Shulgin A., Shulgin A. Pikhal. A chemical love story, Transform press, Berkeley, 1995, $978 \mathrm{pp}$.
17.Shulgin A., Shulgin A. Tikhal. The continuation, Transform press, Berkeley, 1997, 804 pp.

18. Kuhlmann T., Gladbach B. Ecstasy, eine Designerdroge der Techno-Szene. Psychiat. Prax., 1996 ; 23 : 266-9.

19.de Mondenard J.P. Dopage aux jeux olympiques, la triche récompensée. Amphora, Paris, 1996, 447 pp.

20.Klein R.F., Sperling A.R., Cooper D.A., Kram T.C. The stereoisomers of 4-methylaminorex. J. Forensic Sci. 1989 ; 34 (4) : 962-979.

21. Young R., Glennon R.A. Cocaine-stimulus generalization to two new designer drugs : methcathinone and 4-methylaminorex. Pharmacol. Biochem. Behav. $1993 ; 45$ : 229-31.

22.Davis F.T., Brewster M.E. A fatality involving U4Euh, a cyclic derivative of phenylpropanolamine. J. Forensic Sci. 1988 ; 33 (2) : 549-533.

23. Brewster M.E., Davis F.T. Appearance of aminorex as a designer analog of methylaminorex. J. Forensic Sci. 1991 ; 36 (2) : 587-592.

24.Nichols D.E. Differences between the mecanism of action of MDMA, MBDB and the classic hallucinogens. Identification of a new therapeutic class : entactogens. J. Psychoactive Drugs 1986 ; (18) 305-13.

25.Gouzoulis-Mayfrank E., Hermle L., Kovar K.A., Sass H. The entactogens : Ecstasy (MDMA), Eve (MDE) and other ring-substituted methamphetamine derivatives. Nervenartz $1996 ; 67: 369-80$.

26.McGuire P., Fahy T. Chronic paranoid psychosis after misuse of MDMA. Br. Med. J. $1991 ; 302: 697$.

27.Henry J.A., Jeffreys K.J., Dawling S. Toxicity and deaths from 3,4 methylenedioxymetamphetamine (ecstasy). Lancet $1992 ; 340: 384-7$.

28.Cox D.E. "Rave" to the grave. Forensic Sci Int. $1993 ; 60$ : 5-6.

29.Martineau C. Consommation d'ecstasy : des effets neuropsychiques délétères à long terme. Quotidien Med. 1999 ; $6573: 11$.

30.Tucker G. Adam and Eve make Love. Hum. Exper. Toxicol. $1996 ; 15: 455-8$.

31.Dowling G.P., McDonough E.T., Bost R.O. Eve and ecstasy : a report of five deaths associated with the use of MDEA and MDMA. JAMA $1987 ; 257$ (12):1615-7.

32.Kintz P., Cirimele V., Jamey C., Tracqui A., Ludes B. Adam et Eve : association fatale. Toxicorama 1997 ; 9 (2) : 83-6.

33. Ghysel M.H., Dupont V., Kintz P., Tracqui A., Pépin G., Tourneau J. De la rave au cauchemar -2-Les décès après consommation d'amphétamines et dérivés. J. Med Lég. Droit Med. 2000 ; à paraître.

34.Ingold R. Rapport à l'observatoire français des drogues et des toxicomanies, 1999.

35.Kaa E. Drug abuse in western Denmark during the eighties. I. drugs of abuse. Forensic Sci. Int. $1992 ; 55$ (1) : 67-74.

36.Simonsen K.W., Kaa E., Nielsen E., Rollmann D. Street drugs in Denmark 1995-1998. 37" TIAFT triennial meeting, Krakow (Poland) 5-9 sept. 1999.

37.Cami Morell J. Sintesis clandestina de drogas : pasado, pre sente y futuro. Med. Clin. (Barc) 1990 ; 95 (9) : 344-7.

38.de la Fuente I., Rodriguez M.A., Vicente J, Sanchez J., Barrio G. Epidemiologia del consumo de drogas de diseno en Espana. Med. Clin. (Barc) 1997 ; 108 (2) : 54-61. 
39.Baudot P., Dayre S., Laval R., Viriot M.L., Carré M.C. "Ecstasy" (MDMA) et dérivés. Ann. Fals. Exp. Chim. 1998 ; 91 (942) : 81-100.

40.Ghysel M.H., Salvadore O., Colas P., Girard J., Samat J., Sentenac J.C., Molinaro R., Pépin G., Ricordel I., Tourneau J. De la rave au cauchemar-1-Les amphétamines et dérivés. Que consomme-t-on? J. Med Lég. Droit Med. 2000 ; à paraître.

41.Giroud C., Augsburger M., Sadeghipour F., Varesio E., Veuthey J.L., Rivier L., Mangin P. Ecstasy et "raves" en Suisse romande. Toxicorama 1997 ; 9 (2) : 113-122.

42. Malaurie G. La déferlante des drogues chimiques. Le Nouvel Observateur. $2000 ; 1837: 10-20$.

43. Kua E.H. Designer drugs. The ecstasy and the agony. Singapore Med J. 1997 ; 38 (6) : 1.

44. Cimbura G. PMA deaths in Ontario. Can. Med. Assoc. I. $1974 ; 110: 1263-7$.

45.de Boer D., Egberts T., Maes R.A.A. Para-methylthioamphétamine, a new designer drug of abuse. Pharm. World Sci. $1999 ; 21$ (1) : 47-8.

46. King L.A., Poortman-van den Meer A.J., Huizer H. 1-phenylethylamines : a new series of illicit drugs. Forensic Sci. Int. $1996: 77$ (3) : 141-9.

47.Meyer E., van Bocxlaer J., Lamber W., Thienpont L., de Leenheer A. $\alpha$-phenylethylamine identified in judicial samples. Forensic sci. Int. 1995 ; 76 (3) :159-60.

48.Massetti J. ß-phenethylamines in suspected methamphetamine samples. IAFS $15^{\text {th }}$ triennial meeting, San Fransisco (USA) 22-28 août1999.

49.Renton R.J., Cowie J.S., Oon M.C.H. A study of the precursors, intermediates and reaction by-products in the synthesis of 3,4-MDMA and its application to forensic drug analysis. Forensic Sci. Int. $1993 ; 60: 189-202$.

50.Dal Cason T.A. The characterisation of some 3,4-MDA analogs. J. Forensic Sci.1989 ; 34 : 928-61.

51.Dal Cason T.A. An evaluation of the potential for clandestine manufacture of MDA analogs and homologs. J. Forensic Sci. 1990 ; 35 : 675-697.

52. Verweij A.M. Contaminants in illegal amphetamine. Basic contaminant in drug market 3,4-MDMA. Arch. Kriminol. $1991 ; 188(5-6): 154-8$.

53.Forbes I.J., Kirkbride K.P. The origin of alkenes in illicit amphetamines : an examination of the illicitsynthesis of phenyl-2-propanone. J. Forensic Sci. $1992 ; 37$ (5) : $1311-8$

54. Verweij A.M. Contamination in illegal amphetamine. Hydrastinine as a contaminant in 3,4-MDMA. Arch. Kriminol. $1991 ; 188$ (1-2) : 54-7.

55.Angelos S.A. Methamphetamine by the Birch reaction. IAFS $15^{\text {"1 }}$ triennial meeting, San Fransisco (USA) 22-28 août 1999.

56. Willers-Russo L.J. Three fatalities involving phosphine gas, produced as a result of methamphetamine maznufacturing. J. Forensic Sci. 1999 ; 44 (3) : 647-52.

57.Ely R.A., McGrath D.C. Lithium-ammonia reduction of eohedrine to methamphetamine : an unusual clandestine synthesis. J. forensic Sci. 1990 ; 35 (3) : 720-3.

58.Julian A.E. Microcrystalline identification of drugs of abuse : psychedelic amphetamines. J. Forensic Sci. 1990 ; 35 (4) : 821-30.
59. Skinner H.F. Methamphetamine synthesis via reductive alkylation hydrogenolysis of $\mathrm{P}-2 \mathrm{P}$ with $\mathrm{n}$-benzylmethamine. Fortensic Sci. Int. $1993 ; 60: 155-62$.

60. Yap A.T.W., Chen S.X, Lee T.K. An approach to the analysis of methamphetamine in illicit "ice" seizures. IAFS $15^{\text {"t }}$ triennial meeting, San Fransisco (USA) 22-28 août 1999.

61. Sievert H.J.P. Determination of amphetamine and methamphetamine enantiomers by chiral derivatization and GC/MS as a test for an automated sample preparation system. Chirality $1994 ; 6: 295-301$.

62.Jirovsky D., Lemr K., Sevcik J., Smysl B., Stransky Z. Methamphetamine-properties and analytical methods of enantiomer determination. Forensic Sci. Int. 1998 ; 96 : 61-70.

63. Kobayashi K., Kanmori T., Iwata Y., Kishi T. Determination of methamphetamine and its related compounds enatiomer ratois by HPLC with circular dichroism detection. IAFS $15^{\text {th }}$ triennial meeting, San Fransisco (USA) 22-28 août 1999.

64. Bailey K. Legault D. The use of carbon-13 NMR spectra in the identification and authentication of monomethoxyamphetamines and dimethoxyamphetamines. J. Forensic Sci. $1981 ; 26$ (1) : 27-34.

65. Schäfer T., Schönberger T. Computer assisted analysis and comparison of synthetic drug substances containing tablets by NMR spectroscopy. IAFS $15^{\text {th }}$ triennial meeting, San Fransisco (USA) 22-28 août 1999.

66.Lee G.S.H, Craig D.C., Kamali G.S., Dawson M., Conn C., Robertson J., Wilson M.A. Analysis of 3,4-MDMA in Ecstasy tablets by ${ }^{13} \mathrm{C}$ solid state NMR spectroscopy. J. Forensic Sci. 1999 ; 44 (4) : 761-71.

67.Frost M., Köhler H., Blaschke G. Analysis of Ecstasy by capillary electrophoresis. Int. J. Legal Med. 1996 ; 109 : 53-7.

68.Thormann W., Wey A., Lurie H., Gerber H., Byland C., Malik N., Hochmeister M., Gehrig C. Capillary electrophoresis in clinical and forensic analysis : recent advances and breakthrough to routine applications. Electrophoresis $1999 ; 20: 3203-36$.

69.Dirinck I., Praisler M., Van Bocxlaer J., De Leenheer A., Massart D.L. Comparison between automated knowledgebased systems identifying and classifying amphetamine analogues using vapor-phase FTIR spectra and mass spectra. $37^{\text {th }}$ TIAFT triennial meeting, Krakow (Poland) 5-9 sept. 1999.

70.Sondermann N., Kovar K.A. Identification of ecstasy in complex matrices using near-infrared spectroscopy. Forensic Sci. Int. 1999 ; 102 : 133-47.

71. Sondermann N., Kovar K.A. Screening experiments of ecstasy street samples using near-infrared spectroscopy. Forensic Sci. Int. $1999 ; 106$ : 147-56.

72. Vinner E., Dehon B., Ghysel M.H., Lhermitte M. Les psychostimulants. In : Alcool, médicaments, stupéfiants et conduite automobile. Mura P., Elsevier, Paris, 1999, pp. $97-128$ 